\title{
A SIMPLE PROOF OF A THEOREM OF SENSITIVITY
}

\author{
Jorge Iglesias* and Aldo Portela* \\ Universidad de La República. Facultad de Ingenieria. IMERL \\ Julio Herrera y Reissig 565. C.P. 11300 \\ Montevideo, Uruguay
}

(Communicated by )

\begin{abstract}
We prove that every transitive and non minimal semigroup with dense minimal points is sensitive. When the system is almost open, we obtain a generalization of this result.
\end{abstract}

\section{INTRODUCTION.}

The dynamical system that we will consider is formally defined as a triplet $(\mathcal{S}, X, \Phi)$ where $\mathcal{S}$ is a topological semigroup and $\Phi: \mathcal{S} \times X \rightarrow X$ is a continuous function with $\Phi\left(s_{1}, \Phi\left(s_{2}, x\right)\right)=\Phi\left(s_{1} s_{2}, x\right)$ for all $s_{1}, s_{2} \in \mathcal{S}$ and for all $x \in X$ and $X$ is a metric space. The map $\Phi$ is called an action of $\mathcal{S}$ on $X$. We will denote $\Phi(s, x)$ by $\Phi_{s}(x)$. Devaney defines a function to be chaotic if it satisfies the following three conditions: transitivity, having dense set of periodic points and sensitive dependence on initial conditions. In BBCDS it was proved that the two first conditions imply the last one. This result was generalized in AAK, by changing density of periodic points by density of minimal points. In $\mathrm{G}$. this result was generalized when $\mathcal{S}$ is a group, in [KM] was generalized to $C$-semi groups and in [D] for a continuous semi-flow and $X$ being a Polish space. The goal of this paper is to generalize the result in [KM], giving a very simple proof. In [GRSS it is possible to find other results about sensitivity.

1.1. Basic definitions. Let $(\mathcal{S}, X, \Phi)$ be a dynamical system. For any $x \in X$ we define the orbit of $x$ as $O(x)=\left\{\Phi_{s}(x): s \in \mathcal{S}\right\}$. A non-empty set $Y \subset X$, is minimal if $\overline{O(y)}=Y$ for any $y \in Y$. A point $x \in X$ is minimal if the set $\overline{O(x)}$ is a compact and a minimal set. We denote by $\mathcal{M}$ the set of minimal points. We say that the dynamical system $(\mathcal{S}, X, \Phi)$ is minimal if there exists a minimal point $x \in X$ such that $\overline{O(x)}=X$.

The dynamical system $(\mathcal{S}, X, \Phi)$ is point transitive $(\mathrm{PT})$ if there exists $x \in X$ such that $\overline{O(x)}=X$, it is topologically transitive (TT) if given two opene (= open and non-empty) sets $U, V \subset X$ there exists $s \in \mathcal{S}$ such that $\Phi_{s}(U) \cap V \neq \emptyset$ and it is densely point transitive (DPT) if there exists a dense set $Y \subset X$ of transitive points. Denote by $\operatorname{Trans}(X)$ the set of transitive points. If $X$ is a Polish space (i.e. separable completely metrizable topological space) then TT implies DPT.

We say that a dynamical system $(\mathcal{S}, X, \Phi)$ is sensitive if there exists $\varepsilon>0$ such that for all $x \in X$ and for all $\delta>0$ there exists $y \in B(x, \delta)$ and $s \in \mathcal{S}$ such that

2020 Mathematics Subject Classification. Primary: 37B05; Secondary: 20M30 .

Key words and phrases. Semigroups actions, Transitivity, sensitivity. 
$d\left(\Phi_{s}(x), \Phi_{s}(y)\right)>\varepsilon$.

We will now state our main result:

Theorem 1.1. Assume that the dynamical system $(\mathcal{S}, X, \Phi)$ is TT, non-minimal and $\mathcal{M}$ is a dense set. Then $(\mathcal{S}, X, \Phi)$ is sensitive.

We say that the map $\Phi_{s}, s \in \mathcal{S}$, is almost open if $\operatorname{int}\left(\Phi_{s}(U)\right)(\operatorname{int}(W)$ denotes interior of $\mathrm{W})$ is non-empty whenever $U$ is opene. The dynamical system $(\mathcal{S}, X, \Phi)$ is almost open if $\Phi_{s}$ is almost open for any $s \in \mathcal{S}$. The almost open dynamical systems include dynamical systems whose elements are open functions or homeomorphisms. When $X$ is a manifold and the elements of the system are $C^{1}$ functions, the set of critical points can be with non-empty interior. But in this case, an open set can not be mapped at a point.

When the system is almost open we obtain a generalization of the Theorem 1.1

Denote by $\mathcal{M}^{-1}=\cup_{s \in \mathcal{S}} \Phi_{s}^{-1}(\mathcal{M})$.

Theorem 1.2. Assume that the dynamical system $(\mathcal{S}, X, \Phi)$ is almost open, TT and non-minimal. If $\mathcal{M}^{-1}$ is dense then $(\mathcal{S}, X, \Phi)$ is sensitive.

In section 3 we construct an example that shows that the above result no longer holds without the hypothesis that $(\mathcal{S}, X, \Phi)$ is almost open.

\section{Proof of Theorems 1.1 and 1.2}

For the proof of the main theorems we need the following lemma:

Lemma 2.1. Let $x$ be a minimal point and $U_{x}$ a neighbourhood of $x$. Then there exist $s_{1}, \ldots, s_{n_{0}} \in \mathcal{S}$ such that for all $z \in \overline{O(x)}$ there exists $i \in\left\{1, \ldots, n_{0}\right\}$ with $\Phi_{s_{i}}(z) \in U_{x}$.

Proof. As $x$ is a minimal point, given $z \in \overline{O(x)}$ there exist $s_{z} \in \mathcal{S}$ such that $\Phi_{s_{z}}(z) \in U_{x}$. By continuity of $\Phi_{s_{z}}$ there exists a neighbourhood $V_{z}$ of $z$ such that $\Phi_{s_{z}}\left(V_{z}\right) \subset U_{x}$. As $\overline{O(x)} \subset \cup_{z \in \overline{O(x)}} V_{z}$, by the compactness of $\overline{O(x)}$, there exist $z_{1}, \ldots, z_{n_{0}} \in \overline{O(x)}$ such that $\overline{O(x)} \subset V_{z_{1}} \cup \cdots \cup V_{z_{n_{0}}}$. Then, the functions $\Phi_{s_{z_{1}}}, \ldots, \Phi_{s_{z_{n_{0}}}}$ satisfy the lemma.

\section{Proof of Theorem 1.1;}

As the dynamical system $(\mathcal{S}, X, \Phi)$ is non-minimal and $\mathcal{M}$ is dense there exists $M_{1}$ and $M_{2}$ minimal sets with $M_{1} \cap M_{2}=\emptyset$. Let $8 \delta=d\left(M_{1}, M_{2}\right)$. We will show that the dynamical system $(\mathcal{S}, X, \Phi)$ has sensitive dependence on initial conditions with sensitivity constant $\delta$. Let $x \in X$ and $U_{x}$ a neighbourhood of $x$ with $U_{x} \subset B(x, 2 \delta)$. Note that $d\left(x, M_{1}\right) \geq 4 \delta$ or $d\left(x, M_{2}\right) \geq 4 \delta$. Suppose that $d\left(x, M_{1}\right) \geq 4 \delta$. As $\mathcal{M}$ is dense there exists $y \in \mathcal{M} \cap U_{x}$ with $B(y, \delta) \subset B(x, 2 \delta)$. By Lemma 2.1 there exist $\Phi_{s_{1}}, \ldots, \Phi_{s_{n_{0}}}$ such that for all $z \in \overline{O(y)}$ there exists $i \in\left\{1, \ldots, n_{0}\right\}$ with $\Phi_{s_{i}}(z) \in B(y, \delta)$.

Let $W$ be a neighbourhood of $M_{1}$ such that

$$
d\left(\Phi_{s_{i}}(W), B(x, 2 \delta)\right) \geq \delta \text { for any } i \in\left\{1, \ldots, n_{0}\right\} .
$$

As the dynamical system $(\mathcal{S}, X, \Phi)$ is TT then there exists $w \in U_{x}$ and $\Phi_{s}$ such that $\Phi_{s}(w) \in W$. Let $i \in\left\{1, \ldots, n_{0}\right\}$ such that $\Phi_{s_{i}} \Phi_{s}(y) \in B(y, \delta) \subset B(x, 2 \delta)$. As $\Phi_{s_{i}} \Phi_{s}(w) \in \Phi_{s_{i}}(W)$ and $d\left(\Phi_{s_{i}}(W), B(x, 2 \delta)\right) \geq \delta$, then $d\left(\Phi_{s_{i}} \Phi_{s}(y), \Phi_{s_{i}} \Phi_{s}(w)\right) \geq \delta$. 
When $X$ is a Polish space and TT, Theorem 1.1 generalizes the main result given in $[\mathrm{KM}]$.

The Lemma 2.2 shows that PT and $\mathcal{M}$ dense implies TT, so we obtain the following corollary:

Corollary 1. Assume that the dynamical system $(\mathcal{S}, X, \Phi)$ is PT and non-minimal. If $\mathcal{M}$ is dense then $(\mathcal{S}, X, \Phi)$ is sensitive.

Lemma 2.2. Assume that $(\mathcal{S}, X, \Phi)$ is $P T$ and $\mathcal{M}$ is dense. Then $(\mathcal{S}, X, \Phi)$ is TT.

Proof. Let $U$ and $V$ be opene sets and $z \in X$ such that $\overline{O(z)}=X$. Then there exist $\Phi_{s}, \Phi_{s_{1}}$ and $W_{z}$ a neighbourhood of $z$ such that $\Phi_{s}\left(W_{z}\right) \subset U$ and $\Phi_{s_{1}}\left(W_{z}\right) \subset V$. As $\mathcal{M}$ is dense there exists $y \in \mathcal{M} \cap W_{z}$. Then $\Phi_{s}(y) \in U$ and $\Phi_{s_{1}}(y) \in V$. As $y$ is a minimal point then there exists $\Phi_{s_{2}}$ such that $\Phi_{s_{2}} \Phi_{s}(y)$ is close enough to $\Phi_{s_{1}}(y)$ so that $\Phi_{s_{2}} \Phi_{s}(y) \in V$. Then $\Phi_{s_{2}}(U) \cap V \neq \emptyset$.

2.1. Proof of Theorem 1.2. Note that $\mathcal{M}$ is a disjoint union of minimal sets. The proof will be divided into two cases.

Case (a): $\mathcal{M}$ is a minimal set $M$. Let $x_{0} \in X \backslash M$ and $4 \delta=d\left(x_{0}, M\right)$. We will show that the dynamical system $(\mathcal{S}, X, \Phi)$ has sensitive dependence on initial conditions with sensitivity constant $\delta$.

Let $x \in X$ and $U$ be an open set with $x \in U$. As $\mathcal{M}^{-1}$ is dense there exists $y \in U$ and $\Phi_{s}$ such that $\Phi_{s}(y) \in M$. Let $W_{y}$ be a neighborhood of $y, W_{y} \subset U$, such that $d\left(x_{0}, \Phi_{s}\left(W_{y}\right)\right) \geq 3 \delta$.

Since the dynamical system $(\mathcal{S}, X, \Phi)$ is almost open, there exists a open set $V \subset \Phi_{s}\left(W_{y}\right)$. As the system is TT there exist $w \in V$ and $\Phi_{s_{1}}$ such that $\Phi_{s_{1}}(w) \in$ $B\left(x_{0}, \delta\right)$. Note that $w=\Phi_{s}(z)$ for some $z \in W_{y} \subset U$ and therefore $\Phi_{s_{1}}(w)=$ $\Phi_{s_{1}} \Phi_{s}(z) \in B\left(x_{0}, \delta\right)$. As $\Phi_{s_{1}} \Phi_{s}(y) \in M$, then we have that $y, z \in U$ and $d\left(\Phi_{s_{1}} \Phi_{s}(y), \Phi_{s_{1}} \Phi_{s}(z)\right) \geq \delta$.

Case (b): There exist $M_{1}, M_{2}$ minimal sets included in $\mathcal{M}$ with $M_{1} \neq M_{2}$. Let $8 \delta=d\left(M_{1}, M_{2}\right), x \in X$ and $U$ be an open set with $x \in U$. As $\mathcal{M}^{-1}$ is dense there exist $y \in U, \Phi_{s}$ and a minimal set $M$ such that $\Phi_{s}(y) \in M$. Note that $d\left(\Phi_{s}(y), M_{1}\right) \geq 4 \delta$ or $d\left(\Phi_{s}(y), M_{2}\right) \geq 4 \delta$. Suppose that $d\left(\Phi_{s}(y), M_{1}\right) \geq 4 \delta$.

Given $B\left(\Phi_{s}(y), \delta\right)$, by Lemma 2.1 there exist $\Phi_{s_{1}}, \ldots, \Phi_{s_{n_{0}}}$ such that for all $z \in \overline{O\left(\Phi_{s}(y)\right)}$ there exists $i \in\left\{1, \ldots, n_{0}\right\}$ with $\Phi_{s_{i}}(z) \in B\left(\Phi_{s}(y), \delta\right)$. Let $W$ a neighbourhood of $M_{1}$ such that

$$
d\left(\Phi_{s_{i}}(W), B\left(\Phi_{s}(y), \delta\right)\right) \geq 2 \delta \text { for any } i \in\left\{1, \ldots, n_{0}\right\} .
$$

Let $W_{y}$ be a neighborhood of $y, W_{y} \subset U$, such that $\left.\Phi_{s}\left(W_{y}\right)\right) \subset B\left(\Phi_{s}(y), \delta\right)$.

Since the dynamical system $(\mathcal{S}, X, \Phi)$ is almost open, there exists an opene set $V \subset \Phi_{s}\left(W_{y}\right)$. As the dynamical system is TT, there exists $w \in V$ and $\Phi_{r}$ such that $\Phi_{r}(w) \in W$. Note that $w=\Phi_{s}(z)$ for some $z \in W_{y} \subset U$ and $\Phi_{r}(w)=\Phi_{r} \Phi_{s}(z) \in$ $W$. Let $i \in\left\{1, \ldots, n_{0}\right\}$ be such that $\Phi_{s_{i}} \Phi_{r} \Phi_{s}(y) \in B\left(\Phi_{s}(y), \delta\right)$. As $\Phi_{s_{i}} \Phi_{r} \Phi_{s}(z) \in$ $\Phi_{s_{i}}(W)$ and $d\left(\Phi_{s_{i}}(W), B\left(\Phi_{s}(y), \delta\right)\right) \geq 2 \delta$, then $d\left(\Phi_{s_{i}} \Phi_{r} \Phi_{s}(z), \Phi_{s_{i}} \Phi_{r} \Phi_{s}(y)\right) \geq \delta$ and we are done.

\section{ExAmple .}

Let $f_{1}, f_{2}, f_{3}:[0,1] \rightarrow[0,1]$ be as in Figure 1 and $\mathcal{S}$ the free semigroup generated by three elements $a, b$ and $c$. We consider the action $\Phi$ generated by $\Phi_{a}=f_{1}$, 


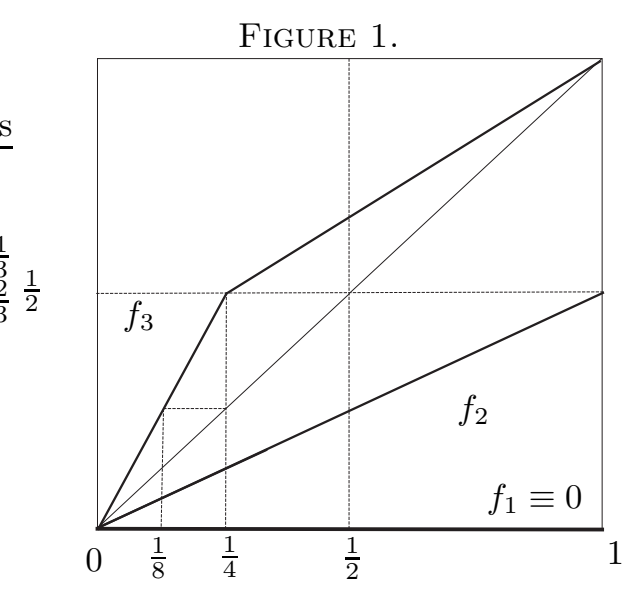

$\Phi_{b}=f_{2}$ and $\Phi_{c}=f_{3}$. Given $s \in \mathcal{S}$, let $A_{s}=\left\{x \in(0,1):\right.$ there exists $\left.\Phi_{s}^{\prime}(x)\right\}$. Note that $A_{s}^{c}$ is a finite set.

As $\left.f_{3}\right|_{\left[0, \frac{1}{8}\right]}=f_{2}^{-1}$ then $f_{3} \circ f_{2}=f_{2} \circ f_{3}=I d$ in $\left[0, \frac{1}{8}\right]$. Since $\left.f_{i}^{\prime}\right|_{\left(\frac{1}{4}, 1\right)}<1$, for all $i=1,2,3$. It is not hard to prove that, for any $s \in \mathcal{S}$ and $x \in A_{s} \cap\left(\frac{1}{2}, 1\right), \Phi_{s}^{\prime}(x)<1$ (see [IP] ). Let $I \subset\left(\frac{1}{2}, 1\right)$ an interval and $s \in \mathcal{S}$. As $A_{s}^{c}$ is a finite set then

$$
\left|\Phi_{s}(I)\right|=\int_{I \cap A_{s}} \Phi_{s}^{\prime}(x) d x .
$$

So, we conclude that $\left|\Phi_{s}(I)\right| \leq|I|$. Therefore the dynamical system $(\mathcal{S}, X, \Phi)$ is not sensitive.

Now we will prove that for all $x \in(0,1], \overline{O(x)}=[0,1]$.

Let $x \in(0,1]$ and suppose that $\overline{O(x)} \neq[0,1]$. Let $I$ be a connected component of $[0,1] \backslash \overline{O(x)}$ with maximum length. It is easy to prove that $f_{3}^{-1}(I)$ or $f_{2}^{-1}(I)$ or $\left(f_{i} f_{j}\right)^{-1}(I)$ with $i, j \in\{2,3\}$ has greater length than $I$, which is a contradiction. Therefore $\overline{O(x)}=[0,1]$.

As $\mathcal{M}=\{0\}$ and $f_{1}^{-1}(0)=[0,1]=X$, therefore $\mathcal{M}^{-1}$ is dense. So $(\mathcal{S}, X, \Phi)$ is TT and non-minimal, the set $\mathcal{M}^{-1}$ is dense, and $(\mathcal{S}, X, \Phi)$ is non-sensitive.

\section{REFERENCES}

[AAK] E. Akin, J. Auslander and K. Berg, When is a transitive map chaotic?, in: Conference in Ergodyc Theory and Probability (V. Bergelson, K. March and J. Rosenblatt, eds.), de Gruyter, Berlin, (1996).

[BBCDS] Banks, J.; Brooks, J.; Cairns, G.; Davis, G.; Stacey, P., On Devaney's definition of chaos, Amer. Math. Monthly, 99 (1992), no. 4, 332-334.

[D] Dai, Xiongping. , Chaotic dynamics of continuous-time topological semi-flows on Polish spaces, J. Differential Equations, 258 (2015) , no. 8, 2794-2805.

[GRSS] F. H. Ghane, E. Rezaale, M. Saleh and A. Sarizadeh, Sensitivity of iterated function systems, J. Math. Anal. Appl. 469 (2019), no. 2, 493-503.

[G] E. Glasner, Ergodic Theory via Joinings. Math. Surveys and Monographs, vol. 101. Am. Math. Soc., Providence (2003).

[IP] Iglesias, J., Portela, A, Almost open semigroup actions, Semigroup Forum 98 (2019), no. 2, 261-270.

[KM] Kontorovich, E., Megrelishvili, M, A note on sensitivity of semigroup actions, Semigroup Forum, 76 (2008), 133-141. 
J. Iglesias, Universidad de la República. Facultad de Ingenieria. imerl. Julio Herrera y Reissig 565. C.P. 11300. Montevideo, Uruguay

Email address: jorgei@fing.edu.uy

A. Portela, Universidad de la República. Facultad de Ingenieria. imerl. Julio Herrera y Reissig 565. C.P. 11300. Montevideo, Uruguay

Email address: aldo@fing.edu.uy 